\title{
Nonlocal and integral conditions problems for a multi-term fractional-order differential equation
}

\author{
A.M.A. El-Sayed and E.O. Bin-Taher
}


Vol. 15 (2014), No. 2, pp. 439-446

\title{
NONLOCAL AND INTEGRAL CONDITIONS PROBLEMS FOR A MULTI-TERM FRACTIONAL-ORDER DIFFERENTIAL EQUATION
}

\author{
A. M. A. EL-SAYED AND E. O. BIN-TAHER
}

Received 03 May, 2012

\begin{abstract}
In this paper we study the existence of solution for a multi-term arbitrary (fractional) order differential equation with some nonlocal and integral conditions.
\end{abstract}

2010 Mathematics Subject Classification: 34B10; 26A33

Keywords: fractional calculus, nonlocal condition, integral condition, fractional differential equation

\section{INTRODUCTION}

Problems with non-local conditions have been extensively studied by several authors in the last two decades. The reader is referred to $[1,2,5]$ and references therein. In this work we study the existence of at least one absolutely continuous solution $x \in A C[0,1]$ for the nonlocal problem of the arbitrary (fractional) order differential equation

$$
x^{\prime}(t)=f\left(t, D^{\alpha_{1}} x(t), D^{\alpha_{2}} x(t), \cdots, D^{\alpha_{n}} x(t)\right), \alpha_{i} \in(0,1) \text {, a.e. } t \in(0,1)
$$

with the nonlocal condition

$$
\sum_{k=1}^{m} a_{k} x\left(\tau_{k}\right)=\beta \sum_{j=1}^{p} b_{j} x\left(\eta_{j}\right)
$$

where $a_{k}, b_{j}>0, \tau_{k} \in(a, c), \eta_{j} \in(d, b), 0<a<c \leq d<b<1$, $\sum_{k=1}^{m} a_{k} \neq \beta \sum_{j=1}^{p} b_{j}$ and $\beta$ is parameter.

As an application, we deduce the existence of solution for the nonlocal problem of the differential equation (1.1) with the nonlocal condition

$$
\sum_{k=1}^{m} a_{k} x\left(\tau_{k}\right)=0, \quad \tau_{k} \in(a, c) \subset(0,1),
$$


and the nonlocal integral conditions

$$
\int_{a}^{c} x(s) d s=\beta \int_{d}^{b} x(s) d s, 0<a<c \leq d<b<1,
$$

and

$$
\int_{a}^{c} x(s) d s=0,(a, c) \subset(0,1) .
$$

are also considered.

\section{PRELIMINARIES}

Let $L^{1}(I)$ denotes the class of Lebesgue integrable functions on the interval $I=[0,1]$,where $0 \leq a<b<\infty$ and let $\Gamma($.$) denotes the gamma function. Recall$ that the operator $T$ is compact if it is continuous and maps bounded sets into relatively compact ones. The set of all compact operators from the subspace $U \subset X$ into the Banach space $X$ is denoted by $C(U, X)$.

Definition 1. The fractional-order integral of the function $f \in L_{1}[a, b]$ of order $\beta>0$ is defined by (see [7])

$$
I_{a}^{\beta} f(t)=\int_{a}^{t} \frac{(t-s)^{\beta-1}}{\Gamma(\beta)} f(s) d s .
$$

Definition 2. The Riemann-Liouville fractional-order derivative of $f(t)$ of order $\alpha \in(0,1)$ is defined as (see [6] and [7])

$$
D_{a}^{\alpha} f(t)=\frac{d}{d t} \int_{a}^{t} \frac{(t-s)^{-\alpha}}{\Gamma(1-\alpha)} f(s) d s .
$$

The following theorems will be needed.

Theorem 1 (Schauder fixed point theorem [3]). Let $E$ be a Banach space and $Q$ be a convex subset of $E$, and $T: Q \longrightarrow Q$ is compact, continuous map, Then $T$ has at least one fixed point in $Q$.

Theorem 2 (Kolmogorov compactness criterion [4]). Let $\Omega \subseteq L^{p}(0,1), 1 \leq p<$ $\infty$. If

(i) $\Omega$ is bounded in $L^{p}(0,1)$, and

(ii) $u_{h} \rightarrow u$ as $h \rightarrow 0$ uniformly with respect to $u \in \Omega$, then $\Omega$ is relatively compact in $L^{p}(0,1)$, where

$$
u_{h}(t)=\frac{1}{h} \int_{t}^{t+h} u(s) d s .
$$




\section{MAIN RESULTS}

Consider firstly the fractional-order functional integral equation

$$
y(t)=f\left(t, I^{1-\alpha_{1}} y(t), \cdots, I^{1-\alpha_{n}} y(t)\right),
$$

Definition 3. The function $y$ is called a solution of the fractional-order functional integral equation (3.1), if $y \in L_{1}[0,1]$ and satisfies (3.1).

Consider the following assumption:

(i) $f:[0,1] \times R_{n} \rightarrow R$ be a function with the following properties:

(a) for each $t \in[0,1], f(t,$.$) is continuous,$

(b) for each $x \in R_{n}, f(., x)$ is measurable,

(ii) there exist an integral function $a, a \in L_{1}[0,1]$ and constants $q_{i}>0, i=1,2$, such that

$$
|f(t, x)| \leq a(t)+\sum_{i=1}^{n} q_{i}\left|x_{i}\right|, \text { for each } t \in[0,1], x \in R_{n},
$$

Theorem 3. Let the assumptions (i) and (ii) be satisfied.

$$
\text { If } \quad \sum_{i=1}^{n} \frac{q_{i}}{\Gamma\left(2-\alpha_{i}\right)}<1,
$$

then the fractional-order functional integral equation (3.1) has at least one solution $y \in L_{1}[0,1]$, where

$$
r \leq \frac{\|a\|}{1-\sum_{i=1}^{n} \frac{q_{i}}{\Gamma\left(2-\alpha_{i}\right)}}
$$

Proof. Define the operator $T$ associated with equation (3.1) by

$$
T y(t)=f\left(t, I^{1-\alpha_{1}} y(t), \cdots, I^{1-\alpha_{n}} y(t)\right)
$$

Let $B_{r}=\left\{y \in L_{1}(I):\|y\|<r, r>0\right\}$ and let $y$ be an arbitrary element in $B_{r}$. Then from the assumptions (i) and (ii), we obtain

$$
\begin{aligned}
\|T y\|_{L_{1}} & =\int_{0}^{1}|T y(t)| d t \\
& \leq \int_{0}^{1}\left|f\left(t, I^{1-\alpha_{1}} y(t), \cdots, I^{1-\alpha_{n}} y(t)\right)\right| d t \\
& \leq \int_{0}^{1}|a(t)| d t+\sum_{i=1}^{n} q_{i} \int_{0}^{1} \int_{0}^{t} \frac{(t-s)^{-\alpha_{i}}}{\Gamma\left(1-\alpha_{i}\right)}|y(s)| d s d t \\
& \leq\|a\|+\sum_{i=1}^{n} q_{i} \int_{0}^{1} \int_{s}^{1} \frac{(t-s)^{-\alpha_{i}}}{\Gamma\left(1-\alpha_{i}\right)} d t|y(s)| d s
\end{aligned}
$$




$$
\begin{aligned}
& \leq\|a\|+\sum_{i=1}^{n} q_{i} \int_{0}^{1}-\left.\frac{(t-s)^{1-\alpha_{i}}}{\left(1-\alpha_{i}\right) \Gamma\left(1-\alpha_{i}\right)}\right|_{s} ^{1}|y(s)| d s \\
& \leq\|a\|+\sum_{i=1}^{n} q_{i} \int_{0}^{1} \frac{1}{\Gamma\left(2-\alpha_{i}\right)}|y(s)| d s \\
& \leq\|a\|+\sum_{i=1}^{n} \frac{q_{i}}{\Gamma\left(2-\alpha_{i}\right)}\|y\|_{L_{1}} \leq r,
\end{aligned}
$$

which implies that the operator $T$ maps $B_{r}$ into itself.

Assumption (i) implies that $T$ is continuous.

Now, we will show that $T$ is compact, applying Theorem 1. So, let $\Omega$ be a bounded subset of $B_{r}$. Then $T(\Omega)$ is bounded in $L_{1}[0,1]$, i.e. condition (i) of Theorem 2 is satisfied. It remains to show that $(T y)_{h} \rightarrow T y$ in $L_{1}[0,1]$ as $h \rightarrow 0$, uniformly with respect to $T y \in T \Omega$. Now

$$
\begin{aligned}
\left\|(T y)_{h}-T y\right\|= & \int_{0}^{1}\left|(T y)_{h}(t)-(T y)(t)\right| d t \\
= & \int_{0}^{1}\left|\frac{1}{h} \int_{t}^{t+h}(T y)(s) d s-(T y)(t)\right| d t \\
\leq & \int_{0}^{1}\left(\frac{1}{h} \int_{t}^{t+h}|(T y)(s)-(T y)(t)| d s\right) d t \\
\leq & \int_{0}^{1}-\frac{1}{h} \int_{t}^{t+h} \mid f\left(s, I^{1-\alpha_{1}} y(t), \ldots, I^{1-\alpha_{n}} y(t)\right) \\
& -f\left(t, I^{1-\alpha_{1}} y(t), \ldots, I^{1-\alpha_{n}} y(t)\right) \mid d s d t .
\end{aligned}
$$

Now, $y \in \Omega$ implies (by assumption (ii)) that $f \in L_{1}(0,1)$, then

$$
\begin{aligned}
\frac{1}{h}-\int_{t}^{t+h}-\mid f\left(s, I^{1-\alpha_{1}} y(t)\right. & \left., \cdots, I^{1-\alpha_{n}} y(t)\right)- \\
& -f\left(t, I^{1-\alpha_{1}} y(t), \cdots, I^{1-\alpha_{n}} y(t)\right) \mid d s \rightarrow 0
\end{aligned}
$$

Therefore, by Theorem 2, we have that $T(\Omega)$ is relatively compact, that is, $T$ is a compact operator, then the operator $T$ has a fixed point $B_{r}$, which proves the existence of a positive solution $y \in(0,1)$ of equation (3.1).

Theorem 4. Let the assumptions of Theorem 3 be satisfied. Then the nonlocal problem (1.1)- (1.2) has at least one solution $x \in A C[0,1]$. 
Proof. Consider the nonlocal fractional differential equation

$$
x^{\prime}(t)=f\left(t, D^{\alpha_{1}} x(t), D^{\alpha_{2}} x(t), \cdots, D^{\alpha_{n}} x(t)\right), \alpha_{i} \in(0,1), \text { a.e. } t \in(0,1),
$$

$\sum_{k=1}^{m} a_{k} x\left(\tau_{k}\right)=\beta \sum_{j=1}^{p} b_{j} x\left(\eta_{j}\right), \quad a_{k}, b_{j}>0, \tau_{k} \in(0, c), \eta_{j} \in(d, 1), c \leq d$.

Let $y(t)=x^{\prime}(t)$, then

$$
x(t)=x(0)+I y(t)
$$

and $y$ is the solution of the fractional-order integral equation (3.1).

Let $t=\tau_{k}$ in equation (3.3), we get

$$
\begin{gathered}
x\left(\tau_{k}\right)=\int_{0}^{\tau_{k}} y(s) d s+x(0) \\
\sum_{k=1}^{m} a_{k} x\left(\tau_{k}\right)=\sum_{k=1}^{m} a_{k} \int_{0}^{\tau_{k}} y(s) d s+x(0) \sum_{k=1}^{m} a_{k}
\end{gathered}
$$

And let $t=\eta_{j}$ in equation (3.3), we get

$$
\begin{gathered}
x\left(\eta_{j}\right)=\int_{0}^{\eta_{j}} y(s) d s+x(0) \\
\sum_{j=1}^{p} b_{j} x\left(\eta_{j}\right)=\sum_{j=1}^{p} b_{j} \int_{0}^{\eta_{j}} y(s) d s+x(0) \sum_{j=1}^{p} b_{j}
\end{gathered}
$$

From equation (1.2), we get

$$
\sum_{k=1}^{m} a_{k} \int_{0}^{\tau_{k}} y(s) d s+x(0) \sum_{k=1}^{m} a_{k}=\beta \sum_{j=1}^{p} b_{j} \int_{0}^{\eta_{j}} y(s) d s+x(0) \beta \sum_{j=1}^{p} b_{j}
$$

Then we get

$$
x(0)=A\left(\sum_{k=1}^{m} a_{k} \int_{0}^{\tau_{k}} y(s) d s-\beta \sum_{j=1}^{p} b_{j} \int_{0}^{\eta_{j}} y(s) d s\right),
$$

where $A=\left(\beta \sum_{j=1}^{p} b_{j}-\sum_{k=1}^{m} a_{k}\right)^{-1}$

and

$$
x(t)=A\left(\sum_{k=1}^{m} a_{k} \int_{0}^{\tau_{k}} y(s) d s-\beta \sum_{j=1}^{p} b_{j} \int_{0}^{\eta_{j}} y(s) d s\right)+\int_{0}^{t} y(s) d s
$$


which, by Theorem 3, has at least one solution $x \in A C(0,1)$.

Now, from equation (3.4), we have

$$
x(0)=\lim _{t \rightarrow 0^{+}} x(t)=A \sum_{k=1}^{m} a_{k} \int_{0}^{\tau_{k}} y(s) d s-A \beta \sum_{j=1}^{p} b_{j} \int_{0}^{\eta_{j}} y(s) d s .
$$

Also

$$
\begin{aligned}
x(1)= & \lim _{t \rightarrow 1^{-}} x(t)=A \sum_{k=1}^{m} a_{k} \int_{0}^{\tau_{k}} y(s) d s-A \beta \sum_{j=1}^{p} b_{j} \int_{0}^{\eta_{j}} y(s) d s \\
& +\int_{0}^{1} y(s) d s
\end{aligned}
$$

from which we deduce that equation (3.4) has at least one solution $x \in A C[0,1]$. To complete the proof

$$
\begin{gathered}
\frac{d x}{d t}=y(t) \\
D^{\alpha_{i}} x(t)=I^{1-\alpha_{i}} \frac{d}{d t} x(t)=I^{1-\alpha_{i}} y(t)
\end{gathered}
$$

where

$$
x^{\prime}(t)=f\left(t, x(t), D^{\alpha_{1}} x(t), D^{\alpha_{2}} x(t), \cdots, D^{\alpha_{n}} x(t)\right) .
$$

Now letting $\beta=0$ in (1.2), we can easily prove the following theorem.

Theorem 5. Let the assumptions (i) - (ii) be satisfied. Then the nonlocal problem $x^{\prime}(t)=f\left(t, D^{\alpha_{1}} x(t), D^{\alpha_{2}} x(t), \cdots, D^{\alpha_{n}} x(t)\right), \alpha_{i} \in(0,1)$, a.e. $t \in(0,1)$,

$$
\sum_{k=1}^{m} a_{k} x\left(\tau_{k}\right)=0, \tau_{k} \in(a, c) \subset(0,1) .
$$

has at least one solution $x \in A C[0,1]$ represented by

$$
x(t)=A \sum_{k=1}^{m} a_{k} \int_{0}^{\tau_{k}} y(s) d s-\int_{0}^{t} y(s) d s, \text { where } A=\left(\sum_{k=1}^{m} a_{k}\right)^{-1} .
$$

\section{NONLOCAL INTEGRAL CONDITION}

Let $x \in A C[0,1]$ be the solution of the nonlocal problem (1.1-1.2).

Let $a_{k}=\tau_{k}-\tau_{k-1}, \quad t_{k} \in\left(\tau_{k-1}, \tau_{k}\right), a=\tau_{0}<\tau_{1}<\tau_{2}, \ldots<\tau_{m}=c$ and $b_{j}=$ $\eta_{j}-\eta_{j-1}, \quad t_{j} \in\left(\eta_{j-1}, \eta_{j}\right), d=\eta_{0}<\eta_{1}<\eta_{2}, \ldots<\eta_{p}=b$ then the nonlocal condition (1.2) will be

$$
\sum_{k=1}^{m}\left(\tau_{k}-\tau_{k-1}\right) x\left(t_{k}\right)=\beta \sum_{j=1}^{p}\left(\eta_{j}-\eta_{j-1}\right) x\left(t_{j}\right) .
$$


From the continuity of the solution $x$ of the nonlocal problem (1.1-1.2) we can obtain

$$
\lim _{m \rightarrow \infty} \sum_{k=1}^{m}\left(\tau_{k}-\tau_{k-1}\right) x\left(t_{k}\right)=\beta \lim _{p \rightarrow \infty} \sum_{j=1}^{p}\left(\eta_{j}-\eta_{j-1}\right) x\left(t_{j}\right) .
$$

and the nonlocal condition (1.2) is transformed to the integral one

$$
\int_{a}^{c} x(s) d s=\beta \int_{d}^{b} x(s) d s .
$$

Also from the continuity of the function $I y(t)$, where $y$ is the solution of the functional integral equation (3.1), we deduce that the solution (3.4) will be

$$
\begin{aligned}
x(t)= & (\beta(b-d)-(c-a))^{-1}\left(\int_{a}^{c} \int_{0}^{s} y(\theta) d \theta d s-\beta \int_{d}^{b} \int_{0}^{s} y(\theta) d \theta d s\right) \\
& +\int_{0}^{t} y(s) d s .
\end{aligned}
$$

Now, we have the following theorem.

Theorem 6. Let the assumptions of Theorem 4 be satisfied. Then there exist at least one solution $x \in A C[0,1]$ of the nonlocal problem with integral condition,

$$
\begin{aligned}
& x^{\prime}(t)=f\left(t, D^{\alpha_{1}} x(t), D^{\alpha_{2}} x(t), \cdots, D^{\alpha_{n}} x(t)\right), \alpha_{i} \in(0,1), \text { a.e. } t \in(0,1), \\
& \int_{a}^{c} x(s) d s=\beta \int_{d}^{b} y(s) d s, 0 \leq a<c \leq d<b \leq 1, \beta(b-d) \neq(c-a) .
\end{aligned}
$$

Letting $\beta=0$ in (4.1), we can easily prove the following corollary.

Corollary 1. Let the assumptions (i) - (ii) be satisfied. Then the nonlocal problem

$$
\begin{gathered}
x^{\prime}(t)=f\left(t, D^{\alpha_{1}} x(t), D^{\alpha_{2}} x(t), \cdots, D^{\alpha_{n}} x(t)\right), \alpha_{i} \in(0,1), \text { a.e. } t \in(0,1), \\
\int_{a}^{c} x(s) d s=0, \quad(a, c) \subset(0,1),
\end{gathered}
$$

has at least one solution $x \in A C[0,1]$ represented by

$$
x(t)=\int_{0}^{t} y(s) d s-(c-a)^{-1} \int_{a}^{c} \int_{0}^{s} y(\theta) d \theta d s .
$$




\section{REFERENCES}

[1] A. Boucherif, "First-order differential inclusions with nonlocal initial conditions," Appl. Math. Lett., vol. 15, no. 4, pp. 409-414, 2002.

[2] A. Boucherif, "Semilinear evolution inclusions with nonlocal conditions," Appl. Math. Lett., vol. 22, no. 8, pp. 1145-1149, 2009.

[3] K. Deimling, Nonlinear functional analysis. Berlin: Springer-Verlag, 1985.

[4] J. Dugundji and A. Granas, Fixed point theory. Monografie Matematyczne, Tom 61. Warszawa: Panstwowe Wydawnictwo Naukowe (PWN - Polish Scientific Publishers), 1982, vol. I.

[5] A. El-Sayed and S. El-Salam, "On the stability of a fractional-order differential equation with nonlocal initial condition,” Electron. J. Qual. Theory Differ. Equ., vol. 2008, p. 8, 2008.

[6] I. Podlubny and A. EL-Sayed, On two definitions of fractional calculus. Slovak Academy of Science-Institute of Experimental phys., 1996.

[7] I. Podlubny, Fractional differential equations. An introduction to fractional derivatives, fractional differential equations, to methods of their solution and some of their applications, ser. Mathematics in Science and Engineering. San Diego: CA: Academic Press, 1999, vol. 198.

Authors' addresses

A. M. A. El-Sayed

Alexandria University, Faculty of Science, Alexandria, Egypt

E-mail address: amasayedehotmail.com

\section{E. O. Bin-Taher}

Hadhramout Univeristy of Science and Technology, Faculty of Science, Hadhramout, Yemen

E-mail address: ebt samsam@yahoo.com 